\title{
Politique
}

\section{L'informatisation de la société : une réflexion critique autour de quelques ouvrages récents}

\section{Johanne Bergeron}

Volume 1, numéro 2, automne 1982

Les médias et les pouvoirs

URI : https://id.erudit.org/iderudit/040407ar

DOI : https://doi.org/10.7202/040407ar

Aller au sommaire du numéro

Éditeur(s)

Société québécoise de science politique

ISSN

0711-608X (numérique)

Découvrir la revue

Citer cet article

Bergeron, J. (1982). L'informatisation de la société : une réflexion critique autour de quelques ouvrages récents. Politique, 1(2), 153-165.

https://doi.org/10.7202/040407ar d'utilisation que vous pouvez consulter en ligne.

https://apropos.erudit.org/fr/usagers/politique-dutilisation/ 


\title{
Synthèse bibliographique
}

\section{L'informatisation de la société: une réflexion critique autour de quelques ouvrages récents}

\author{
par \\ Johanne Bergeron \\ Université de Montréal
}

S'il est un thème aujourd'hui à la mode, c'est bien celui des techniques nouvelles, de la télématique, du processus d'informatisation de la société. Non seulement informaticiens et autres technologues s'affairent à en multiplier les applications à des domaines sans cesse élargis, mais politicologues et sociologues sont entrés dans la ronde pour soumettre à l'analyse les enjeux socio-politiques sous-jacents à ce processus. Il est certain que l'introduction massive dans un espace social de l'ordinateur, associé aux télécommunications (ensemble qu'on appelle la télématique), comporte des enjeux et des conséquences qu'il appartient au sociologue d'analyser. Si, à l'évidence, l'informatique envahit progressivement tous les aspects de la vie sociale et individuelle, cette expansion pose en des termes nouveaux la question de son impact social. Ces conséquences ne sont plus limitées aux relations de travail ou à la gestion d'une entreprise 
ou d'une organisation particulière; c'est bien plutôt l'ensemble des rapports et des pouvoirs d'une société qui sont susceptibles d'être modifiés par ces technologies. L'intérêt sociologique et politique de ce thème paraît ainsi évident.

L'informatisation est donc devenu un concept à la mode. Depuis une dizaine d'années, on ne compte plus les ouvrages sur la technique et l'informatique, qui couvrent la gamme des possibles idéologiques: homme et technique ou technique et société, apologétique ou sceptique, le discours apparaît cependant remarquablement superficiel. Malgré cette production volumineuse de travaux, ce thème de recherche demeure largement sous-développé. Ce secteur de la littérature des sciences sociales se caractérise par une confusion remarquable: entre l'utopie de la machine intelligente et pensante, l'outil à tout faire porteur de pouvoir potentiellement totalitaire, les essais philosophiques sur la technique, l'analyse politique et l'approche journalistique, se manifestent un enchevêtrement des problématiques, une incapacité à formuler clairement les questions, les problèmes et leurs réponses.

J'ai retenu quelques ouvrages sociologiques ou politiques récents qui ont abordé cette question de l'informatisation, dont j'aimerais rendre compte. Ils constituent une bonne illustration de l'état et de la tendance des travaux en cette matière.

On doit le concept de «télématique» à $S$. Nora et $A$. Minc (L'informatisation de la société, Paris, La documentation française, 1978), dans un rapport publié à la demande du président de la République française. Ce rapport vise à «faire progresser la réflexion sur les moyens de conduire l'informatisation de la société » et à apporter des recommandations pour une éventuelle politique gouvernementale dans ce domaine. À ce mandat général, les auteurs ont répondu en examinant les enjeux sociaux, politiques et économiques de l'informatisation et en brossant un tableau d'ensemble de la situation française. La 
télématique y apparaît comme un facteur de transformation de l'organisation économique et sociale; la société de demain sera une société de l'information, et l'État doit intervenir dès maintenant pour orienter le processus d'informatisation dans un sens positif.

Le rapport Nora-Minc a le mérite essentiel de mettre en lumière que la télématique, en envahissant l'espace social, est susceptible de bouleverser le système nerveux de la société dans son ensemble; en effet, contrairement aux innovations technologiques du passé (telles l'électricité ou la machine à vapeur, par exemple), la télématique véhicule non pas un courant inerte mais de l'information, c'est-à-dire du pouvoir. Elle dispose ainsi d'un pouvoir structurant sur les multiples aspects de la vie sociale, économique, politique et culturelle, qui appellent, en raison de leur importance, une intervention de l'État.

Les auteurs se penchent sur chacun de ces aspects, élaborant un scénario de développement comportant dangers et défis potentiels. Au niveau économique, l'informatisation de la société est au cœur de la crise puisqu'elle peut, selon les stratégies adoptées, l'aggraver ou la résoudre. Ainsi, la télématique apportera un gain considérable de productivité mais aggravera le chômage à court terme; par contre, ce gain de productivité améliorera la position concurrentielle de l'économie nationale, ce qui suscitera de nouveaux débouchés et la résorbtion du chômage. Le retour à cette position d'équilibre, condition de la croissance, est facilité par une politique d' «incitations actives » de la part de l'État qui doit ainsi doser son intervention entre une consolidation des secteurs de pointe, compétitifs, et des transferts vers les secteurs moins compétitifs et les consommateurs. La croissance passe ainsi par la télématique.

Au niveau social, la «nouvelle informatique» accroîtra la transparence entre les groupes sociaux, la vulnérabilité des grandes organisations, la mobilité entre les professions; elle permet- 
tra la décentralisation et même l'autonomie des unités de base de toute organisation, allègera la structure des organisations et améliorera leur efficacité. Dans la mesure où l'État est capable d'orienter les évolutions sociales et d'équilibrer les pouvoirs, il semble que la démocratie passe aussi par l'informatisation... En outre, la télématique peut, politiquement, déplacer les enjeux de souveraineté; il importe que l'État national puisse résister aux pressions d'État ou de groupes étrangers, dans un objectif d'indépendance nationale. Enfin, elle transformera les traditions et le modèle culturels.

Pour les auteurs, l'enjeu fondamental de l'introduction massive de la télématique est d'ordre économique: orientée par une stratégie étatique cohérente, elle est un facteur de résorbtion de la crise, bien que ses effets, parce que enjeu de pouvoir, débordent l'aspect purement économique. Cette partie prospectiviste du rapport constitue celle, sans doute en raison de la conjoncture, dont les idées se sont le plus répandu. En fait, cette vision du processus d'informatisation comme panacée aux difficultés du monde moderne, repose sur une conception morale du social: harmonisation des contraintes, des libertés, des aspirations des groupes et des citoyens en vue du projet collectif. De même, l'État se présente comme un arbitre bienveillant et neutre, au-dessus des classes: seul dépositaire de l'information appropriée, l'État peut «préserver l'avenir de la société » (p. 122), favoriser le développement national; «la société et l'État non seulement se supportent, mais se fabriquent mutuellement» (p. 123).

Si je me suis attardée assez longuement au rapport NoraMinc, c'est qu'il a constitué le point de départ de tout un courant d'analyse qui s'en est plus ou moins largement inspiré. Il faut voir que ce travail a le mérite d'avoir opéré un important renversement de perspective théorique: à une problématique surtout juridique qui voit dans l'ordinateur une menace au 
moins potentielle aux libertés individuelles, succède une conception plus économiste qui conçoit l'informatique comme un moyen privilégié d'assurer la croissance et, ainsi, de résorber la crise qui affecte les sociétés occidentales depuis trop longtemps déjà. De même, le débat sur les enjeux sociaux est lancé. L'intérêt majeur du rapport Nora-Minc est d'aborder les aspects techniques et économiques de l'informatisation, en les liant à une interrogation sur le sens de l'évolution sociale.

Ainsi, J. Attali (La nouvelle économie française, Paris, Flammarion, 1978) estime que le développement des réseaux informatiques constitue la voie de sortie la plus probable de la crise. La demande de biens de consommation étant saturée, il faut générer de nouvelles demandes pour relancer la production. Les technologies de l'information joueraient ainsi le rôle qu'ont joué après la guerre l'automobile et les biens ménagers dans la croissance de la production. Au niveau social, l'auteur constate qu'historiquement cette mise en place de nouvelles technologies commercialisées s'est accompagnée de la «marchandisation » des services et des relations humaines; aujourd'hui, l'informatisation se traduira également par la substitution d'une marchandise à la relation humaine, surtout dans les secteurs de la santé, de l'éducation, et de la culture. La vision de la société informatisée véhiculée ici est fortement négative: il s'agit d'une société d'auto-surveillance, totalitaire, normalisée, marchande, où les rapports humains sont évacués. Seul l'avènement parallèle d'une «société socialiste relationnelle» (p. 193) mise en place par l'État permettra d'échapper à cette fatalité puisqu'elle met l'accent sur les réseaux informationnels conçus comme moyens de communication, qui fourniront «à chaque individu les moyens d'une création autonome, éphémère ou durable» ( $\mathrm{p}$. 193).

Par ailleurs, après une analyse fouillée des manifestations et des causes de la crise et après un examen tout aussi sérieux 
des politiques économiques de sortie de crise, J.H. Lorenzi, O. Pastré et J. Toledano (La crise $d u X X^{e}$ siècle, Paris, Economica, 1981) suggèrent que les blocages structurels de l'économie ne pourront être surmontés que par l'émergence et le développement de lieux d'accumulation nouveaux, de nouvelles activités productives, qui permettront de faire apparaître à la fois des gains de productivité et une norme de consommation renouvelée. Or les auteurs soutiennent que la filière industrielle des composants électroniques est susceptible de remplir ce rôle, en raison de son insertion à la fois dans le domaine de la production (biens intermédiaires) et dans celui de la consommation (produits finis). Le développement de cette filière implique un accroissement de sa part de marché, ce qui a comme conséquence l'informatisation et l'automation sociales. Les pertes d'emplois qui en résultent seront compensés par les gains de productivité générés. En ce sens, le développement des technologies informatiques représente la solution globale et à long terme à la crise, par la croissance structurelle ainsi mise en œuvre.

En fait, on peut constater que les tenants de la conception de l'informatisation comme moyen structurel de sortie de crise amènent des arguments semblables. Malgré les pertes d'emplois occasionnés par l'introduction des machines, la reprise est possible par la relance de la production et de la consommation des biens de capital, intermédiaires et des produits finis que génère cette industrie. Les gains de productivité dégagés sont alors susceptibles de contrer et d'absorber à moyen terme, dans une mesure suffisante, les pertes d'emplois constatés à court terme. Autrement dit, la filière électronique est une industrie industrialisante.

C'est là un argument que l'on entend souvent. Cette approche a certes le mérite de rappeler qu'au-delà de son apparence magique et des discours idéologiques, l'informatique est 
d'abord un secteur de la production et de la consommation. Dépassant le niveau du discours, on pourrait cependant s'attendre à ce que la thèse soit mieux étayée. Or il n'en est rien. La «thèse » est laissée sous forme d'hypothèse que l'avenir, on l'espère, se chargera de vérifier positivement, à la condition bien sûr que l'intervention de l'État ait lieu dans le sens et selon le dosage adéquat. Notamment, le chômage massif prévu, effectivement concevable au plan théorique, est peu discuté; il m'apparaît trop simple et étonnamment utopique de penser que ces pertes d'emplois seront automatiquement compensées à moyen terme par l'émergence de gains de productivité qui susciteront de nouveaux débouchés et des activités nouvelles. Même si une référence à l'histoire d'après-guerre est soulevée (production de masse d'automobiles et d'appareils ménagers), encore faudrait-il démontrer la relation causale postulée entre production de biens nouveaux et prospérité. Cette relation est loin d'être évidente: a-t-on même déjà vu dans l'histoire du capitalisme les périodes de crise se résorber par la simple production, induite d'ailleurs par l'État, de biens nouveaux?

On pourrait aussi discuter de la conception de l'État véhiculée ici, intimement liée à une vision du social. Paradoxalement, chez des auteurs de tendance marxiste, on retrouve une vision libérale de l'État, c'est-à-dire un État neutre, informé, au-dessus des classes. La mâtrrise par l'État du processus d'informatisation est en effet souhaitable, parce seule son intervention est susceptible d'orienter l'intégration des machines dans le sens de la plus grande utilité sociale. L'État se porte garant d'une bonne intégration sociale et d'une utilisation adéquate des machines. On retrouve ici la thèse implicite du conditionnement du progrès social par la technique et l'économique: une société outillée saura mieux résoudre ses difficultés. L'État devient en ce sens, selon l'expression de J. Ellul (L'illusion politique, Paris, Livre de Poche, 1977), un simple «agent techni- 
que», voué à la promotion d'une efficacité maximale définie en termes d'impératifs techniques et économiques fondamentaux. L'informatisation de la société exprime ainsi un choix économique: l'informatique, les télécommunications et les activités qui en découlent apparaissent comme un des rares secteurs, par leur potentiel de développement rapide, qui permettent la poursuite de la croissance.

Une autre tendance importante du discours sur l'informatisation favorise et magnifie à la fois le phénomène et ses conséquences sociales. Il s'agit d'une conception essentiellement justificatrice et réductrice qui présente l'informatique comme forme inévitable et absolue de progrès technique et social.

Un des représentants actuels et actifs de ce courant, B. Lussato (Le défi informatique, Paris, Fayard/Sélect, 1981), cherche à mettre en valeur les applications les plus spectaculaires de l'informatique et propose une stratégie d'informatisation basée sur la promotion des petits ensembles. C'est moins la connaissance du processus qui est proposée dans cet ouvrage que son spectacle. En outre, les effets de l'informatisation sont magnifiés: rien de moins qu'une "révolution" par le progrès, qui touchera tous les aspects de la vie humaine, est attendue, dans le sens de l'harmonisation sociale, de l'intégration, de la réification des rapports sociaux. "Notre avenir, clame la page couverture, dépend de la révolution informatique. »

Cette révolution informatique, selon Lussato, trouve son ennemi non pas tant dans la machine que dans le système de gestion et les hommes qui s'en servent. L'auteur propose d'inverser le processus de centralisation qui implique l'utilisation d'une informatique lourde et centralisée (ce que l'auteur appelle le "grand chaudron» et ce que Nora-Minc ont appelé la télématique), au profit des petits ordinateurs privés et des systèmes autonomes (les «petits chaudrons»). Seule la privatique, qui propose la multiplication des petits ordinateurs à fonctions 
limitées, permet d'envisager l'avènement d'une société à la fois techniquement moderne et à dimension humaine, bref conviviale. Car l'homme est, et doit être, la mesure de toute chose, y compris des machines qui le servent. L'auteur soutient que le «petit chaudron» saura enrichir les possibilités créatrices de l'homme, sa sensibilité, son intelligence: «c'est l'homme luimême qui demeurera le pôle essentiel » (308).

Si Lussato a le mérite de soumettre à l'interrogation critique le processus de centralisation par l'informatique, ses arguments à l'appui de la privatique sont, si l'on considère que l'auteur enseigne au Conservatoire national des arts et métiers français, étonnamment pauvres. Le scénario manque de consistance: si la décentralisation doit mener à une société humaniste et conviviale, on ne sait rien sur la voie de transition vers ce type de société. On ne voit pas non plus la relation absolue que postule l'auteur entre ce type de société et de rapports sociaux et l'introduction des petits ordinateurs individuels. En fait, le contexte social est totalement évacué de l'analyse; l'informatique est considérée en elle-même indépendamment des rapports sociaux et économiques dans laquelle elle s'inscrit, c'est-à-dire comme un agent autonome de changement détaché du contexte sociétal global qui l'a produit. Enfin, les illustrations des applications presque magiques de la privatique sont de plus empruntées à la prospective: vidéodisque, robot ménager, terminal domestique individuel. Quel bien ne nous veut pas l'industrie de l'électronique! En somme, le discours de Lussato demeure largement superficiel et vide.

Dans la même veine, J.H. Lorenzi et E. Le Boucher (Mémoires volées, Ramsay, 1979) font précéder leur argumentation d'un prologue qui conduit d'emblée le lecteur dans l'univers automatisé et asceptisé de la fin du vingtième siècle où, selon le scénario retenu, le quotidien dans son ensemble est informatisé, programmé, robotisé. L'analyse des auteurs, entre les 
première et troisième parties qui sombrent dans cet imaginaire prospectiviste qui éclaire bien peu les débats, se révèle toutefois plus intéressante lorsqu'elle s'attarde à l'examen de la filière mondiale de l'électronique et des difficultés pour un État national (ici la France) de se définir une politique autonome et cohérente de développement à ce chapitre. Leur analyse conclut à la grande dépendance industrielle de la France en matière informatique, envers les États-Unis surtout.

Au chapitre des conséquences sociales, l'économiste libéral H. Lepage (Autogestion et capitalisme, Masson, 1978) voit dans la micro-informatique et les télécommunications un facteur de décentralisation et de «convivialisation» de la société. Par l'accessibilité de tout individu aux réseaux cablés, favorisant le travail à domicile, la réception des programmes d'enseignement, la communication, la technologie informatique entraîne avec elle une évolution vers les valeurs conviviales. L'auteur reconnaît bien les effets sociaux négatifs produits par la technologie: robotisation de l'individu, déqualification du travail, frustration. Mais ces problèmes seront résolus par la communication électronique qui non seulement mettra fin au mouvement de concentration industrielle au niveau économique mais surtout permettra aux individus de retrouver des modes d'action et de pensée autonomes, antibureaucratiques, personnalisés. Ces nouvelles valeurs issues de la "révolution de la communication électronique » bouleverseront l'ensemble de l'organisation sociale. Ainsi, «loin d'être une mécanisation accrue des rapports humains, [les nouveaux progrès des techniques informatiques] entraînent une multiplication des possibilités d'intervention des hommes sur ce qui les concerne ou leur est familier, que ce soit dans leur vie professionnelle ou dans leur vie de citoyen» (347).

Triste perspective de recherche qu'offre cette revue de littérature!... Si je n'ai certes pas la prétention d'avoir rendu 
compte de l'ensemble des travaux, je crois cependant en avoir saisi les principaux courants. Il s'en dégage notamment une superficialité remarquable qui camoufle mal un manque de connaissance de l'ampleur du problème soulevé, où l'imagination prend trop souvent le pas sur la rigueur analytique.

En fait, notre examen révèle que l'analyse politique de l'informatisation se révèle remarquablement peu fouillée; peu d'auteurs se soucient, au plan théorique, de replacer l'informatisation dans le contexte socio-politique dans lequel elle s'inscrit, s'interdisant par là d'en comprendre l'immersion sociale. Comme le rappelle pertinemment A. Touraine (Pour la sociologie, Paris, Seuil, 1974), on ne peut que rejeter l'alternative qui consiste à présenter la technologie soit comme un agent autonome de changement ou soit, à l'inverse, comme un outil parfaitement malléable (93). En ce sens, il est étonnant que la plupart des ouvrages considérés évacuent cette perspective; et lorsque prise en compte, la vision du futur débouche sur une société post-industrielle apaisée et harmonieuse.

Par ailleurs, on retrouve dans cette littérature une certaine tradition positiviste ou, du moins, techniciste. Le système de représentation véhiculé valorise la technologie informatique au point d'en faire comme l'équivalent d'une nouvelle foi qui donnerait réponse à tout. Cette technologie semble fonctionner en tant qu'idéologie, susceptible de fournir des solutions à la totalité des problèmes qui se posent. Il y a une quinzaine d'années, J. Habermas (La Technique et la science comme idéologie, Paris, Médiations Gallimard, 1973, é.o. all. 1968) élaborait pourtant une critique approfondie de cette conception, en montrant la non-neutralité des techniques.

Plus fondamentalement, enfin, la plupart des ouvrages parlent très peu de ce qui est pour se concentrer surtout sur ce qui devrait être; c'est une analyse prospectiviste qui est proposée par ce courant d'analyse. Des hypothèses - qui ne peu- 
vent que demeurer au stade d'hypothèses — sont avancées pour prévoir les besoins futurs et les caractéristiques de l'informatisation, le visage de cette société informatisée, son impact économique ou social potentiel, les interventions étatiques nécessaires pour orienter le sens du processus et réaliser les équilibres sociaux.

Or on peut se demander dans quelle mesure une argumentation basée sur une vision prospectivisite du futur est susceptible de déboucher sur une analyse rigoureuse et scientifique du phénomène retenu. À leur stade actuel d'évolution, les sciences de la société, et notamment l'économique et la science politique, réussissent mal à proposer une analyse explicative cohérente du passé et du présent; comment pourraient-elles valablement avancer des scénarios pour l'avenir? Cette prétention m’apparaît un abus scientifique et épistémologique important.

Les prospectivistes, rappelle E. Morin (Pour sortir $d u$ vingtième siècle, Paris, Nathan, 1981), supposent le passé connu, le passé également connu et stable, et que sur ces bases l'avenir se forge suivant les tendances dominantes de l'économie, de la science et des techniques. Cette pensée, qualifiée de technobureaucratique, «dans son optimisme débile» (319), croit ainsi qu'elle peut prévoir l'avenir: les facteurs d'évolution sont connus, la causalité est linéaire, donc le futur est prédictible. C'est là une grande erreur, l'évolution sociale n'est ni mécanique ni linéaire.

Il y a toujours, dit encore Morin, un jeu rétroactif entre passé et présent, «où non seulement le passé contribue à la connaissance du présent, ce qui est évident, mais aussi où les expériences du présent contribuent à la connaissance du passé, et par là le transforment» (320). Or la grande illusion consiste à croire que le présent est connu parce qu'il est présent, c'est-à-dire immédiatement vécu; et comme le futur naît du présent, c'est dire la difficulté, sinon l'impossibilité de penser 
le futur social. Je résiste mal à la tentation de reciter Morin: «Une telle conception, de par les incertitudes qu'elle apporte dans l'apparemment assuré, le passé et le présent, semble devoir frapper de nullité toute tentative de prévoir le futur. En fait, elle dévoile la nullité des prospectives et futurologies qui prétendaient se fonder sur le socle du présent » (322).

C'est peut-être là l'origine du malaise ressenti à la lecture de la majorité des ouvrages réalisés sur le thème de l'informatisation de la société. Il conviendrait de repenser ce thème de recherche, sa méthodologie et la problématique qui sous-tend son traitement sociologique et politique. Cela s'impose en raison des enjeux importants qui y sont liés, qui commandent une connaissance plus approfondie et plus rigoureuse de cet objet d'étude. Mais, au fait, l'informatisation peut-elle se conjuguer au présent? L'histoire n'avance pas comme un fleuve, le devenir s'annonce complexe. C'est dire la difficulté que pose, au niveau épistémologique, l'appréhension du phénomène informatique. 